Title Page

- Title: HYPERSEXUALITY AND PATHOLOGICAL GAMBLING IN PARKINSON'S DISEASE: A CROSS-SECTIONAL CASE-CONTROL STUDY

- List of the authors followed by their highest academic degrees (MD, PhD) and their institutional affiliations.

- Corresponding author:

de Chazeron I. (PhD) Univ Clermont 1, UFR Medecine, EA3845, ClermontFerrand, F-63001 France, CHU Clermont-Ferrand, Psychiatry B, ClermontFerrand, F-63003, France

Rue Montalembert

63000 Clermont-Ferrand, France

Phone +33473754580

Fax +33473752129

Email: idechazeron@chu-clermontferrand.fr

○ Other Authors: E-mail:

Llorca P.M. (MD, PhD) Univ Clermont 1, UFR Medecine, EA3845, Clermont-Ferrand, F-63001, France, CHU Clermont-Ferrand, Psychiatry B, Clermont-Ferrand, F-63003, France pmllorca@chu-clermontferrand.fr

Chéreau-Boudet I. (MD) Univ Clermont 1, UFR Medecine, EA3845, CHU Clermont-Ferrand - Psychiatry B, Clermont-Ferrand, F-63001 France ichereau@chu-clermontferrand.fr

Blanc O. Univ Clermont 1, UFR Medecine, EA3845, Clermont-Ferrand, F63001, France, CHU Clermont-Ferrand, Psychiatry B, Clermont-Ferrand, F63003, France oblanc@ @ chu-clermontferrand.fr

Perriot J. (MD) CHU Clermont-Ferrand - Psychiatry B, Clermont-Ferrand, F63001, France Jean.PERRIOT@cg63.fr

Ouchchane L. (PhD) CHU Clermont-Ferrand - Department of Biostatistics, Clermont-Ferrand, F-63001 France, Lemlih.OUCHCHANE@u-clermont1.fr 
Ulla M. (MD) Univ Clermont 1,UFR Medecine, EA3845, Clermont-Ferrand, F63001, France, CHU Clermont-Ferrand, Neurology A, Clermont-Ferrand, F63003, France mulla@ chu-clermontferrand.fr

Debilly B. (MD) Univ Clermont 1, UFR Medecine, EA3845, ClermontFerrand, F-63001, France, CHU Clermont-Ferrand, Neurology A, ClermontFerrand, F-63003, France bdebilly@ chu-clermontferrand.fr

Derost P. (MD) Univ Clermont 1, UFR Medecine, EA3845, ClermontFerrand, F-63001, France, CHU Clermont-Ferrand, Neurology A, ClermontFerrand, F-63003, France pderost@ chu-clermontferrand.fr

Durif F. (MD, PhD) Univ Clermont 1, UFR Medecine, EA3845, ClermontFerrand, F-63001, France, CHU Clermont-Ferrand, Neurology A, ClermontFerrand, F-63003, France fdurif@ chu-clermontferrand.fr

Keywords: Parkinson's disease; Addiction

Financial Disclosure/Conflict of Interest: None

Word count Abstract: 148 / Word count text: 1509 / Tables: 2 / Figures: 0 


\section{HYPERSEXUALITY AND PATHOLOGICAL GAMBLING IN PARKINSON'S DISEASE: A CROSS-SECTIONAL CASE-CONTROL STUDY}

\section{$\underline{\text { Abstract }}$}

Background: Substance and behavioral addictions have already been described separately or in combination in Parkinson's disease (PD). However, no comparisons of the prevalence of addictive behaviors in patients with PD and the general population have been published.

Objective: To compare the prevalence and characteristics of addictions (gambling, hypersexuality, tobacco and alcohol) in PD patients and in a matched, paired sample from the general population.

Method: After matching for age, sex, and complete field questionnaires on addictions, we had 115 data-sets.

Results: No difference was observed between PD and control population concerning pathological gambling $(0.87 \%$ vs. $0.87 \%, \mathrm{p}=0.99)$, tobacco addiction $(1.7 \%$ vs. $1.7 \%, \mathrm{p}=0.99)$ and alcohol dependence $(2.6 \%$ vs. $3.5 \%, \mathrm{p}=0.71)$. The PD group showed two cases of sexual addiction $(1.7 \%$ vs. $0, \mathrm{p}=0.15)$.

Conclusion: Our results indicate that PD patients do not have specific profiles for tobacco or alcohol addiction and pathological gambling compared with the general population. 


\section{Introduction}

Impulse control disorders or addiction behaviors are often reported during the post-treatment evolution of parkinsonian patients: they include pathological gambling (PG) ${ }^{1}$, compulsive shopping ${ }^{2}$, eating disorders ${ }^{3}$ and hypersexuality ${ }^{4}$. The global prevalence of addictive disorders in Parkinson's disease (PD) is estimated to be around $14 \%{ }^{5}$. However, it is unknown whether the prevalence of addictive behaviors in PD differs from that observed in the general population (after controlling for gender and age). In fact, some addictions such as pathological gambling ${ }^{6}$ seem more frequent in Parkinson's disease and others, such as addiction to alcohol and tobacco, less frequent. Many studies have reported a lower prevalence of smoking and alcohol consumption (known as the most common substance addictions) in PD than in general population ${ }^{7-9}$. Based on those observations, we proposed a descriptive epidemiological study to define prevalence and characteristics of addictions (gambling and hypersexuality, tobacco and alcohol) in PD patients compared with a matched paired sample originating from the general population.

\section{Method}

\section{Study Design}

From 1st September 2006 to 31 August 2007, all the consecutive patients with idiopathic PD according to Queen Square Brain Bank criteria ${ }^{10}$ referred as outpatients to Unit of Movement Disorder of Clermont-Ferrand Hospital, France, were screened. Exclusion criteria were atypical Parkinsonism, dementia (Mini-Mental Status Examination (MMSE) <24), deep brain stimulation and inability to complete the survey. During the same period, the control sample was recruited from people who had an appointment for a free health check-up, referred from the National Health System. Exclusion criteria were Parkinsonism, and inability to complete the survey. Each subject from the control group was selected for one-to-one matching on sex 
and birth date (plus-minus 6 years). All subjects gave written consent to participate in this study.

\section{General and Clinical Evaluation}

Socio-demographic data were collected for all participants: age, sex, occupation. For PD patients, information on duration of disease and antiparkinsonian treatment were also collected. All eligible participants completed a survey including: Fagerström Test for Nicotine Dependence (FTND) ${ }^{11}$; Alcohol Use Disorders Identification Test (AUDIT) ${ }^{12}$; South Oaks Gambling Screen (SOGS) ${ }^{13}$; Hypersexuality questionnaire (HS) ${ }^{14}$ which is a shorter and French version of the Sexual Addiction Screening Test (SAST).

\section{Results}

\section{Characteristics of population}

We examined a consecutive series of 159 idiopathic PD subjects and a series of 188 controls. Distribution of missing data in PD patients and controls were not significantly different (Table 1). After screening all the completed filed questionnaires, 139 PD patients were selected and after age- and sex-matching the controls, we obtained 115 patients and controls as detailed in Table 2. No difference in terms of age, sex ratio, situation and occupational class characteristics were found between the two matched groups (Parkinson's and control groups) (Table 1). The mean disease duration of the PD group was 7.4 years (SD 4.0, range 1-20 years), mean Levodopa daily dose was $631 \mathrm{mg} / \mathrm{d}$ (SD 436) and mean dopamine agonist daily dose was $130 \mathrm{mg} / \mathrm{d}$ (SD 168) (calculated on the basis of Levodopa correspondences adapted from Thobois et al. $\left.{ }^{15}\right)$. Ten patients $(8.7 \%)$ were without agonist or L-dopa, 40(34.8\%) with L-dopa alone, 4 (3.5\%) with agonist alone, 61 (53.0\%) with agonist(s) adjunct. 


\section{Addiction characteristics}

Patients with pathological gambling or "at risk gambler"or with sex addiction were not significantly more frequently in the PD group, compared with the control group (Table 2). For tobacco addiction, (classified as non-smoker, low - no dependence, medium dependence) there was no statistically significant difference in patterns between 115 PD patients and paired controls even though there were twice as many smokers in the control population as among the PD patients.

The prevalence of current (referral within previous 12-months) alcohol abstinence among PD patients was nearly twice the prevalence among the paired controls and harmful alcohol use among the control group was significantly more frequent $(\mathrm{p}=0.04)$. Three PD patients and four paired controls had an alcohol dependence (Table 2).

\section{Discussion}

This study is original in comparing addictive profiles in PD patients compared with a gender and age matched general population sample.

In our samples, pathological gambling did not occur more frequently in PD patients than in the general population, in contrast to sexual addiction.

In our sample, the prevalence of "problem gambling" (at risk and pathological) was $13.1 \%$. It was higher than the observations of Crockford et al. $2008^{16}(9.3 \%)$ and Singh et al. $2007^{17}$ $(9.3 \%)$. The rate of pathological gambling was lower than those presented by Voon et al. 2006 ${ }^{18}$, Avanzi et al. $2006^{6}$ and Weintraub et al. $2010^{5}(5.0 \%)$. The discrepancy in results may be related to different methods of evaluating and characterising the gambling.

In our study, identification of pathological gamblers was done using the same screening instrument (SOGS) as the one used by Voon et al. ${ }^{18}$ but with a cut-point different from that used in the original paper of Lesieur and Blume ${ }^{13}$. From a methodological point of view, we 
preferred a more clinically relevant cut-point as suggested by Shaffer and Hall ${ }^{19}$. If we class PD patients scoring $>=3$ as pathological gamblers, as did Voon et al. ${ }^{18}$, compared with $>=5$ as in our study, we find a rate of $2.6 \%$ which is in their described range of $1.7-3.4 \%$ for the prevalence of PG. Avanzi et al. $2006^{6}$, also using the SOGS with a lifetime observation, found a prevalence of PG close to 6\%. The difference could be explained by the period of time explored (lifetime or past-30-day) and also by the sample sizes of the groups studied. Weintraub et al. ${ }^{5}$ used the Massachusetts Gambling Screen instrument that has been reported to be a less suitable measure for screening purposes compared with the SOGS ${ }^{20}$. In the population we studied, the prevalence of PG did not differ between the PD and the control groups. This result contrasts with the literature. In the study of Avanzi et al. ${ }^{6} 0.25 \%$ of the general population was diagnosed to have PG compared with $6.12 \%$ in the PD population. Two possible explanations may have influenced the results in the general population. Firstly, until recently, gambling was generally viewed negatively by Italian citizens and specific anti-gambling programs have been developed in schools ${ }^{21}$. Secondly, in this context, a PG screening procedure was introduced, consisting of an interview by the general practitioner. The low prevalence obtained in this general population may be related to information bias (under-declaration). Our PG prevalence in general population is closer to the one described in Europe ${ }^{22}$, accepted as being around $1 \%$ to $2 \%$.

The prevalence of pathological hypersexuality observed in our PD patients group is $1.7 \%$. Voon et al. $2006^{18}$ suggested a current prevalence of $2.0 \%$, Weintraub et al. ${ }^{5}$ around $3.5 \%$. This last prevalence differs from ours probably because the screening instrument used (MIDI) needs at least two positive responses to diagnose compulsive sexual behavior compared with five in our study. Singh et al. in $2007{ }^{17}$ described a prevalence of around 10\%. However, in their study, all of the patients included had been exposed to dopamine agonists, which was not the case in our study. In the control group, we found no cases of pathological hypersexuality, 
but it is generally acknowledged that the highest level of sexual addiction is established between the ages of 36 and 50 years ${ }^{23}$.

Because tobacco smokers have a well-documented lower incidence of PD compared with the general population, tobacco dependency in PD patients is not studied. First of all, looking at the prevalence of smoking, we found a 50\% higher prevalence of smoking in the control group than in patients with PD. This has previously also been observed in other studies 24,25 . This proportion of smokers is lower $(2.6 \%, \mathrm{n}=3)$ but close to recent data on a large sample $\left(3.8 \%{ }^{5}\right)$. Tobacco dependence seemed not to differ between control and PD groups. Significantly fewer PD patients drink alcohol compared with the control population. Our proportion of fully abstinent PD patients (32.2\%) is similar to that described in the study of Hernan et al. ${ }^{9}$. PD patients who were screened positively for harmful drinking were significantly fewer than in the control population. However, no difference was observed concerning patients with alcohol dependence between the two groups of subjects.

Taken together, these data confirm the lower prevalence of tobacco smokers and drinkers in PD patients compared with the controls but they also suggest a close prevalence of alcohol and tobacco dependence in the two populations. This comparison should be considered with caution in view of the small numbers of cases and further work is needed to determine whether changes in alcohol and tobacco dependency are specific for PD. We must notice that co-dependencies exist: one case of dopamine dysregulation syndrome (DDS) and tobacco medium dependency and at risk gambler; one case of DDS and at risk gambler and one case of DDS and alcohol (Alcohol dependent).

The most surprising result of this work is that the prevalence of pathological/at-risk gambling is not higher in PD population that in the control and also that alcohol and tobacco dependence did not differ between the two groups, although further studies are needed to confirm this finding. Beyond elucidating some underlying causes of addiction, this study also 
highlights the lack of addictions-specific assessment instruments. The challenge for the future is to detect patients through a good routine screening instrument which could save cost and time.

\section{Acknowledgement}

This study was sponsored by a Hospital Program for Clinical Research (Local funding),

Clermont-Ferrand University Hospital-FRANCE. We gratefully acknowledge the contribution of the people who were involved in recruitment and particularly Mrs Christine Delaigue and Mr Didier Delhaye.

\section{References}

1. Molina JA, Sainz-Artiga MJ, Fraile A, et al. Pathologic gambling in Parkinson's disease: a behavioral manifestation of pharmacologic treatment? Mov Disord 2000;15(5):869-872.

2. Giladi N, Weitzman N, Schreiber S, Shabtai H, Peretz C. New onset heightened interest or drive for gambling, shopping, eating or sexual activity in patients with Parkinson's disease: the role of dopamine agonist treatment and age at motor symptoms onset. J Psychopharmacol 2007;21(5):501-506.

3. Nirenberg MJ, Waters C. Compulsive eating and weight gain related to dopamine agonist use. Mov Disord 2006;21(4):524-529.

4. Vogel HP, Schiffter R. Hypersexuality--a complication of dopaminergic therapy in Parkinson's disease. Pharmacopsychiatria 1983;16(4):107-110.

5. Weintraub D, Koester J, Potenza MN, et al. Impulse control disorders in Parkinson disease: a cross-sectional study of 3090 patients. Arch Neurol 2010;67(5):589-595.

6. Avanzi M, Baratti M, Cabrini S, Uber E, Brighetti G, Bonfa F. Prevalence of pathological gambling in patients with Parkinson's disease. Mov Disord 2006;21(12):2068-2072.

7. Allam MF, Campbell MJ, Del Castillo AS, Fernandez-Crehuet Navajas R. Parkinson's disease protects against smoking? Behav Neurol 2004;15(3-4):65-71.

8. Evans AH, Lawrence AD, Potts J, et al. Relationship between impulsive sensation seeking traits, smoking, alcohol and caffeine intake, and Parkinson's disease. J Neurol Neurosurg Psychiatry 2006;77(3):317-321.

9. Hernan MA, Chen H, Schwarzschild MA, Ascherio A. Alcohol consumption and the incidence of Parkinson's disease. Ann Neurol 2003;54(2):170-175.

10. Gibb WR, Lees AJ. The relevance of the Lewy body to the pathogenesis of idiopathic Parkinson's disease. J Neurol Neurosurg Psychiatry 1988;51(6):745-752.

11. Fagerstrom KO, Heatherton TF, Kozlowski LT. Nicotine addiction and its assessment. Ear Nose Throat J 1990;69(11):763-765. 
12. Saunders JB, Aasland OG, Babor TF, de la Fuente JR, Grant M. Development of the Alcohol Use Disorders Identification Test (AUDIT): WHO Collaborative Project on Early Detection of Persons with Harmful Alcohol Consumption--II. Addiction 1993;88(6):791-804.

13. Lesieur HR, Blume SB. The South Oaks Gambling Screen (SOGS): a new instrument for the identification of pathological gamblers. Am J Psychiatry 1987;144(9):11841188.

14. Carnes PJ. Out of the Shadows.Understanding sexual addiction. 3rd Revised edition ed. Center City, 2001.

15. Thobois S. Proposed dose equivalence for rapid switch between dopamine receptor agonists in Parkinson's disease: a review of the literature. Clin Ther 2006;28(1):1-12.

16. Crockford D, Quickfall J, Currie S, Furtado S, Suchowersky O, El-Guebaly N. Prevalence of problem and pathological gambling in Parkinson's disease. J Gambl Stud 2008;24(4):411-422.

17. Singh A, Kandimala G, Dewey RB, Jr., O'Suilleabhain P. Risk factors for pathologic gambling and other compulsions among Parkinson's disease patients taking dopamine agonists. J Clin Neurosci 2007;14(12):1178-1181.

18. Voon V, Hassan K, Zurowski M, et al. Prospective prevalence of pathologic gambling and medication association in Parkinson disease. Neurology 2006;66(11):1750-1752.

19. Shaffer HJ, Hall MN. Estimating prevalence of adolescent gambling disorders: A quantitative synthesis and guide toward standard gambling nomenclature. J Gambling Stud 1996;12:193-214.

20. Weinstock J, Whelan JP, Meyers AW, McCausland C. The performance of two pathological gambling screens in college students. Assessment 2007;14(4):399-407.

21. Meyer G, Hayer T, Griffiths M. Problem Gambling in Europe: Challenges, Prevention, and Interventions. New York: Springer, 2009.

22. Biganzoli A, Capelli M, Capitanucci D, Smaniotto R, Alippi M. [An investigation into pathological gambling. Epidemiological study of gambling attitudes and prevalence of pathological gambling in the Pavia province (Italy)]. 2004.

23. Lindau ST, Schumm P, Laumann E, Levinson W, O'Muircheartaigh C, Waite L. Gender, Age and Health Differences in Sexuality at Older Ages: A National PopulationBased Study. Journal of the American Geriatrics Society 2007;55(4-S3).

24. Alves G, Kurz M, Lie SA, Larsen JP. Cigarette smoking in Parkinson's disease: influence on disease progression. Mov Disord 2004;19(9):1087-1092.

25. Scott WK, Zhang F, Stajich JM, Scott BL, Stacy MA, Vance JM. Family-based casecontrol study of cigarette smoking and Parkinson disease. Neurology 2005;64(3):442447. 
Documentation of Author Roles

de Chazeron I. 1A, 1B, 1C, 2B, 3A

Llorca P.M 1A, 3B

Chéreau-Boudet I. 1A

Blanc O. 2C

Perriot J. 1A, 3B

Ouchchane L. 2A, 2B, 2C

Ulla M. 1C

Debilly B. 1C

Derost P. 1C

Durif F. 1A, 3B 